\title{
Local thermal non-equilibrium effects arising from the injection of a hot fluid into a porous medium
}

\author{
D. ANDREW S. REES ${ }^{1}$, ANDREW P. BASSOM ${ }^{2}$ \\ AND PRADEEP G. SIDDHESHWAR ${ }^{1,3}$ \\ ${ }^{1}$ Department of Mechanical Engineering, University of Bath, Bath BA2 7AY, UK \\ ${ }^{2}$ School of Mathematics and Statistics, University of Western Australia, Crawley, WA 6009, Australia \\ ${ }^{3}$ Department of Mathematics, Bangalore University, Bangalore, India
}

(Received 30 March 2007 and in revised form 2 September 2007)

We examine the effect of local thermal non-equilibrium on the infiltration of a hot fluid into a cold porous medium. The temperature fields of the solid porous matrix and the saturating fluid are governed by separate, but coupled, parabolic equations, forming a system governed by three dimensionless parameters. A scale analysis and numerical simulations are performed to determine the different manners in which the temperature fields evolve in time. These are supplemented by a large-time analysis showing that local thermal equilibrium between the phases is eventually attained. It is found that the thickness of the advancing thermal front is a function of the governing parameters rather than being independent of them. This has the implication that local thermal equilibrium is not equivalent to a single equation formulation of the energy equation as might have been expected. When the velocity of the infiltrating fluid is sufficiently large, the equations reduce to a hyperbolic system and a thermal shock wave is formed within the fluid phase. The strength of the shock decays exponentially with time, but the approach to local thermal equilibrium is slower and is achieved algebraically in time.

\section{Introduction}

Heat and mass transfer in porous media arises in a number of important applications, including thermo-solutal convection in geothermal fields (McKibbin 2005), post-accident heat removal from pebble-bed reactors (Baytas 2003), the disposal of hazardous waste in subsurface salt formations (Schotting \& Landman 2004) and the modelling of convection in the underground storage of $\mathrm{CO}_{2}$ (Ennis-King, Preston \& Paterson 2005). Many studies are concerned, in particular, with the flushing out of contaminants, oil or heat from various types of porous medium. The process of heat extraction from geothermal fields, for instance, is in widespread use around the world for domestic and industrial heating and for the generation of electricity (Barbier 2002). In a hot dry rock formation, water is pumped underground, heated by the rock and then extracted from a production well. On the other hand, extraction of hot water in many geothermal reservoirs depends on the fact that the saturated formation is at high pressure, but, to preserve the pressure, the spent water is reinjected into the formation (Shook 2001) as part of the standard well-management schemes (Stopa \& Wojnarowski 2006). In both these cases, then, and also in those procedures where 
heat either has to be removed or introduced, the common feature is that a fluid with a different temperature is used to cause heat transfer from or to the surrounding solid phase.

Studies such as those of Shook (2001) and Stopa \& Wojnarowski (2006) employ a hyperbolic approximation to the equation of heat transport, assuming that the presence of temperature variations does not affect the velocity of the injected fluid. Both papers extend a much earlier analysis by Bodvarsson (1972); Shook (2001) does this by considering a heterogeneous porous medium, while Stopa \& Wojnarowski (2006) allow the physical properties of the rock and the injected fluid to exhibit realistic variations with temperature. In both cases conduction is neglected as a second-order effect, and the resulting hyperbolic system is solved to determine the detailed thermal front.

However, in both these studies and many others, the temperature fields of the rock and the fluid are assumed to be identical: such a situation is generally known as Local Thermal Equilibrium (LTE). The converse situation is known as Local Thermal Non-Equilibrium (LTNE), and in these cases the solid porous matrix may have a different temperature from that of the saturating fluid, this being understood in terms of averages over representative elementary volumes. Thus hot fluid can flow into a cold, relatively insulating porous matrix, and there will exist a difference in the average local temperature of the two phases which will take time to reduce to values where one could say that the phases are in LTE. When the phases are not in LTE the usual single energy equation should usually be replaced by two, one for each phase. First introduced by Anzelius (1926) and Schumann (1929), these equations use simple linear source/sink terms which allow for the local transfer of heat between the phases. These early equations, which lack diffusion terms, have been used in very many subsequent studies, especially for the modelling of heat exchangers. The standard equations that are now used routinely include diffusion and are those quoted by Nield \& Bejan (2006).

An early study by Combarnous \& Bories (1974) on the the effect of LTNE on nonlinear Darcy-Bénard convection and some more recent papers by Straughan (2006), Baytas (2003), Baytas \& Pop (2002) and Banu \& Rees (2001) on DarcyBénard convection, and Rees (2003) and Rees \& Pop (2000) on boundary layers, represent much of the published work on the effect of LTNE on free convection in porous media: see the detailed review by Rees \& Pop (2005). On the other hand, the literature on forced convective heat transfer is considerably more extensive. Some of the more recent papers have concentrated on the steady introduction of hot fluid into a channel with cold sidewalls, a variant on the classical Graetz problem: see for example, Nield, Kuznetsov \& Xiong (2002) and Nield (1998). This important subject has not yet been the subject of a detailed review.

The chief aim of the present paper is to investigate the detailed effect of LTNE on the propagation of thermal fronts in a one-dimensional porous medium. The fronts are generated by the sudden injection of hot (cold) fluid into a cold (hot) porous medium. Physical properties are kept constant with respect to temperature, the porous medium is assumed to be homogeneous, and buoyancy forces are neglected. In a remarkably early study Anzelius (1926) presented an analysis of the thermal front propagation in the absence of conduction in both phases using the method of characteristics. Burch, Allen \& Peavy (1976) considered a finite slab of porous medium, with application to single blow heating of packed beds, their aim being to determine the time taken to reach a steady temperature. In this case conduction was neglected only in the fluid phase. Toovey \& Dayan (1985) studied an infinite heat reservoir, similar to the 
present situation, and conduction in the fluid phase was also neglected. Kuznetsov (1994) employed a perturbation technique wherein the heat gained by the fluid phase from the solid phase could be neglected. The emphasis of his work was on the decaying solitary wave-like properties of the difference in the temperatures of the two phases. Kuznetsov (1998) then considered the sudden injection of hot fluid into finite two- and three-dimensional regions, expressing the subsequent evolution of the temperature fields analytically. Use was made of the Anzelius-Schumann model but with the inclusion of the diffusion terms in both phases. On the other hand, Swailes $\&$ Potts (2006) studied the injection of a hot compressible gas into a cold porous medium consisting of packed spheres. The thermal field corresponding to the spheres was accounted for by means of a suitably modified form of the Newton's law of cooling boundary condition, relating the local temperature of the gas to heat lost by the spheres to the gas. Thus only one thermal field needs to be employed in such a formulation. Abu-Hijleh, Al-Nimr \& Hader (2004) have made a preliminary study of the present problem, but the focus of their short paper was on the 'thermalization' time, the time taken for LTE to be re-established, and only numerical data of this kind were presented.

In the present paper we consider the the evolution of the temperature fields themselves and find that there are parameter regimes within which thermal shock waves are formed, that these shocks decrease in strength with time, and that they travel faster than the velocity of the injected fluid - it is within this regime that the approach to LTE is very slow indeed. In addition, we discover that there are other parameter regimes wherein the solid phase heats up more rapidly than the fluid phase, despite the fact that the fluid is moving. Thus, the present paper represents a substantial extension of the work of Abu-Hijleh et al. (2004) and Anzelius (1926) by uncovering the detailed behaviour of the thermal fields.

The remainder of the paper develops as follows. Section 2 introduces the governing equations and describes a scale analysis indicating the important parameter regimes. Some detailed numerical solutions are presented in $\S 3$, and these are complemented by a large-time analysis which is outlined in $\S 4$. Here we show, in particular, that the asymptotic solution is in LTE but, unusually, its structure is dependent on the three governing parameters. In $\S 5$ a hyperbolic approximation is made, which corresponds to high flow rates or poor heat transfer between the phases. Laplace Transform solutions are obtained and compared with full numerical simulations of the original parabolic equations. Finally, we offer some comments and discussion in $\S 6$.

\section{Governing equations}

Our study of LTNE effects is conducted using a model in which the semi-infinite expanse of saturated porous medium lies in the region $x^{*} \geqslant 0$. Fluid is supposed to enter at $x^{*}=0$ with a steady uniform flux velocity $v^{*}=(U, 0,0)$ and the system is in thermal equilibrium with both phases taken to have the same temperature $T_{0}$. In addition, we assume that the fluid is incompressible and buoyancy forces are negligible. Suddenly, at time $t^{*}=0$, the temperature of the incoming fluid is changed to $T_{1}$, which may be higher or lower than $T_{0}$, and is maintained thereafter at this new level.

The governing equations for the evolution of the temperature fields of the fluid and solid phases of a rigid porous medium have been described by Nield \& Bejan (2006). If $\varepsilon$ denotes the porosity of the medium, and if subscripts $\mathrm{f}$ and $\mathrm{s}$ attached to variables refer to fluid and solid properties in the obvious way, then the equations 
coupling the temperature fields $T_{\mathrm{f}}$ and $T_{\mathrm{S}}$ are

$$
\begin{aligned}
\varepsilon(\rho c)_{\mathrm{f}} \frac{\partial T_{\mathrm{f}}}{\partial t^{*}}+(\rho c)_{\mathrm{f}} v^{*} \cdot \nabla T_{\mathrm{f}} & =\varepsilon \nabla \cdot\left(k_{\mathrm{f}} \nabla T_{\mathrm{f}}\right)+h\left(T_{\mathrm{S}}-T_{\mathrm{f}}\right), \\
(1-\varepsilon)(\rho c)_{\mathrm{S}} \frac{\partial T_{\mathrm{S}}}{\partial t^{*}} & =(1-\varepsilon) \nabla \cdot\left(k_{\mathrm{S}} \nabla T_{\mathrm{S}}\right)+h\left(T_{\mathrm{f}}-T_{\mathrm{S}}\right) .
\end{aligned}
$$

Furthermore, the fluid density and thermal capacity are given by $\rho$ and $c$ while the thermal conductivity is $k$. It is important to note that $k_{\mathrm{f}}$ and $k_{\mathrm{s}}$ are not the intrinsic conductivities of the fluid and solid phases, respectively, but rather are effective conductivities; see Wakao \& Kaguei (1982) or Vafai \& Amiri (1998).

Within this system the inter-phase heat transfer coefficient $h$ is worthy of especial note. Large values of $h$ imply that LTE is achieved very quickly, by which we mean that the phases tend quickly to the same temperature locally. Conversely, for small values of $h$, little heat is lost from the fluid to the solid, and it takes a relatively long time for the solid phase to attain the same local temperature as the fluid phase; this is precisely LTNE. In general, the value of $h$ depends on many factors including the detailed geometry of the porous medium, the porosity, the flow field and the conductivities and diffusivities of the phases, as reviewed in Rees \& Pop (2005). Recent work by Rees (2007) has drawn attention to the fact that many of the published expressions for $h$ do not yield the correct symmetries in the zero flow limit and, in some cases, are independent of the conductivity of the solid phase. In the present paper we shall assume that it is a constant, which, given that the imposed flow field is uniform and steady, means that our analysis is independent of how $h$ is modelled (unless it is also dependent on the temperature difference between the phases thereby rendering the source/sink terms nonlinear, a scenario which has not been considered). In system (2.1) we follow standard practice and take $T_{\mathrm{f}}$ and $T_{\mathrm{S}}$ to be intrinsic averages of the respective temperature fields. This allows us to set $T_{\mathrm{f}}=T_{\mathrm{S}}=T_{0}$ whenever the boundary of the porous medium is maintained at the temperature $T_{0}$. On the other hand, $v$ is a superficial average, which has important ramifications later.

The problem examined here does not possess an obvious natural length scale $L$ although it does contain the velocity scale, $U$. However, $L$ may be defined in terms of $U$ and the other natural properties of the porous medium and the saturating fluid so that $L=k_{\mathrm{pm}} / U(\rho C)_{\mathrm{f}}$. Then the system (2.1) may be non-dimensionalized using the substitutions

$$
\begin{aligned}
x^{*} & =L x, \quad \boldsymbol{v}^{*}=\frac{k_{\mathrm{pm}}}{L(\rho C)_{\mathrm{f}}} \boldsymbol{v}, \\
t^{*} & =\frac{L^{2}(\rho C)_{\mathrm{pm}}}{k_{\mathrm{pm}}} t, \quad\left(T_{\mathrm{f}}, T_{\mathrm{s}}\right)=T_{0}+\left(T_{1}-T_{0}\right)(\theta, \phi)
\end{aligned}
$$

and where all quantities $\Xi$ associated with the porous medium have been defined according to the weighted arithmetic mean

$$
\Xi_{\mathrm{pm}}=\varepsilon \Xi_{\mathrm{f}}+(1-\varepsilon) \Xi_{\mathrm{s}}
$$

On assuming that variations take place only in the $x$-direction, as the flow is unidirectional, the governing equations become

$$
\begin{gathered}
\left(\frac{\gamma+1}{\gamma+\alpha}\right) \frac{\partial \theta}{\partial t}+\left(\frac{\gamma+1}{\gamma}\right) \frac{\partial \theta}{\partial x}=\frac{\partial^{2} \theta}{\partial x^{2}}+H(\phi-\theta), \\
\alpha\left(\frac{\gamma+1}{\gamma+\alpha}\right) \frac{\partial \phi}{\partial t}=\frac{\partial^{2} \phi}{\partial x^{2}}+H \gamma(\theta-\phi) .
\end{gathered}
$$


The three dimensionless parameters $H, \gamma$ and $\alpha$ are the non-dimensional inter-phase heat transfer parameter, the porosity-modified conductivity ratio and the diffusivity ratio, and are given by

$$
H=\frac{h L^{2}}{\varepsilon k_{\mathrm{f}}}, \quad \gamma=\frac{\varepsilon k_{\mathrm{f}}}{(1-\varepsilon) k_{\mathrm{s}}}, \quad \alpha=\frac{k_{\mathrm{f}}}{(\rho C)_{\mathrm{f}}} \frac{(\rho C)_{\mathrm{s}}}{k_{\mathrm{s}}}=\frac{\alpha_{\mathrm{f}}}{\alpha_{\mathrm{s}}} .
$$

It is worth pointing out that the complicated coefficients in (2.4) have distinctive meanings in terms of ratios of conductivities and diffusivities since

$$
\frac{\gamma+1}{\gamma+\alpha}=\frac{\alpha_{\mathrm{pm}}}{\alpha_{\mathrm{f}}}, \quad \frac{\gamma+1}{\gamma}=\frac{k_{\mathrm{pm}}}{\varepsilon k_{\mathrm{f}}} \quad \text { and } \quad \alpha\left(\frac{\gamma+1}{\gamma+\alpha}\right)=\frac{\alpha_{\mathrm{pm}}}{\alpha_{\mathrm{s}}} .
$$

We also notice that (2.4) simplifies greatly in the LTE $(H \rightarrow \infty)$ limit. When $H$ is asympotically large we have $\theta=\phi(\equiv \Theta$, say) to leading order (so that, equivalently, $\left.\theta-\phi=O\left(H^{-1}\right)\right)$ where

$$
\Theta_{t}+\Theta_{x}=\Theta_{x x} .
$$

Finally, it is important to note that the replacement of the equation (2.7) by the system (2.4) means that the order of the system has been increased from one to two, thereby raising the possibility of oscillatory solutions in time. The analysis of Vadasz (2005) reduces the pair of equations for the respective temperatures to just one, which is second-order in time and fourth-order in space. Conditions are then derived for this system to exhibit under-damped oscillations, but these conditions cannot be met in practice. Therefore the approach to a steady-state solution is always overdamped.

\subsection{Coordinate transformations and scale analysis}

The sudden change in the inlet condition is highly reminiscent of the classical unsteady conduction problem described by Carslaw \& Jaeger (1959) in which one end of a conducting solid at a uniform temperature has its temperature instantaneously raised to a new constant level. In the light of their work, it is natural to make the coordinate transformation

$$
\eta=\frac{x}{2 \sqrt{t}}, \quad \tau=\sqrt{t}
$$

so that equations (2.4) become

$$
\begin{gathered}
\left(\frac{\gamma+1}{\gamma+\alpha}\right) 2 \tau \frac{\partial \theta}{\partial \tau}=\frac{\partial^{2} \theta}{\partial \eta^{2}}+\left[\left(\frac{\gamma+1}{\gamma+\alpha}\right) 2 \eta-\left(\frac{\gamma+1}{\gamma}\right) 2 \tau\right] \frac{\partial \theta}{\partial \eta}+4 \tau^{2} H(\phi-\theta), \\
\alpha\left(\frac{\gamma+1}{\gamma+\alpha}\right) 2 \tau \frac{\partial \phi}{\partial \tau}=\frac{\partial^{2} \phi}{\partial \eta^{2}}+\alpha\left(\frac{\gamma+1}{\gamma+\alpha}\right) 2 \eta \frac{\partial \phi}{\partial \eta}+4 \tau^{2} H \gamma(\theta-\phi) .
\end{gathered}
$$

This system needs to be supplemented by suitable starting and boundary conditions. The initial temperature profiles are given by

$$
\theta=\phi=0 \quad \text { at } \quad t=0,
$$

while the boundary conditions are taken to be

$$
\begin{gathered}
\theta=1, \quad \frac{\partial \phi}{\partial \eta}=0 \quad \text { at } \quad \eta=0 \\
\theta, \phi \longrightarrow 0 \quad \text { as } \quad \eta \longrightarrow \infty .
\end{gathered}
$$

We have chosen to use an insulating condition for the solid phase at $\eta=0$ as it models the situation that occurs in an experiment, such as where hot fluid enters a thin horizontal layer via a narrow slit at $x=0$, in which case the boundary condition 
is a symmetry condition. This condition results in the value of $\phi$ at $\eta=0$ rising towards 1 as $\tau$ increases and as the passage of hot fluid heats up the relatively cold solid by means of the source/sink terms. It might be argued that the setting of $\phi=1$ at $\eta=0$ would be equally appropriate, but this modification affects only the detailed early-time solutions.

Before embarking on a numerical solution of the problem, there is some benefit in considering the expected behaviour of the solutions to system (2.9). It is clear from these equations and the associated boundary conditions that $\theta=O(1)$ and $\phi=0$ when $\tau=0$. If we suppose that both $\alpha$ and $\gamma$ are of roughly unit magnitude, then the two most important quantities in equations (2.9) would seem to be $\tau$ and $\tau^{2} H$. Sufficiently small values of $\tau$ mean that the second term in the coefficient of $\theta_{\eta}$ in equation (2.9a) is negligible at leading order, and then solutions to the full system take the forms

$$
\begin{aligned}
& \theta=\operatorname{erfc} \zeta+\tau\left(\frac{(\gamma+\alpha)(\gamma+1)}{\gamma^{2}}\right)^{1 / 2} \zeta \operatorname{erfc} \zeta+O\left(\tau^{2}\right), \\
& \phi=\frac{H \gamma(\gamma+\alpha)}{(1-\alpha)(\gamma+1)}\left[G(\zeta)-\frac{1}{\sqrt{\alpha}} G(\sqrt{\alpha} \zeta)\right] \tau^{2}+O\left(\tau^{3}\right),
\end{aligned}
$$

where

$$
\zeta \equiv\left(\frac{\gamma+1}{\gamma+\alpha}\right)^{1 / 2} \eta \quad \text { and } \quad G(\zeta) \equiv\left(2 \zeta^{2}+1\right) \operatorname{erfc} \zeta-\frac{2}{\sqrt{\pi}} \zeta \mathrm{e}^{-\zeta^{2}}
$$

It is important to note that we also require $\tau^{2} H \ll 1$ for these results to hold, since otherwise the source/sink terms in (2.9) are no longer negligible at leading order. Indeed, the magnitude of $\tau^{2} H$ determines roughly when $\phi$ makes the transition from being small to becoming of $O(1)$ magnitude; at such times, the coupling terms in (2.9) first allow heat to be transferred between the phases. This allows us to estimate the various transition stages in the evolution of the thermal fields and the details of these are sensitive to the relative magnitudes of $\tau$ and $\tau^{2} H$. When the system is likely to be near to LTE, that is when $H \gg 1$, we have that

$$
\begin{aligned}
\theta \sim \operatorname{erfc} \sqrt{\frac{\gamma+1}{\gamma+\alpha} \eta,} \quad \phi & =O\left(\tau^{2} H\right) & \text { when } & & \tau \ll H^{-1 / 2} \\
\theta, \phi & \sim \operatorname{erfc} \eta & \text { when } & & H^{-1 / 2} \ll \tau \ll 1 \\
\theta, \phi & \sim \frac{1}{2} \operatorname{erfc}(\eta-\tau / 2) & \text { when } & & 1 \ll \tau .
\end{aligned}
$$

Conversely, in the opposite limit $H \ll 1$ and far from LTE, the equivalent results are

$$
\begin{gathered}
\theta \sim \operatorname{erfc} \sqrt{\frac{\gamma+1}{\gamma+\alpha} \eta, \quad \phi=O\left(\tau^{2} H\right) \quad \text { when } \quad \tau \ll 1} \\
\theta \sim \frac{1}{2} \operatorname{erfc} \sqrt{\frac{\gamma+1}{\gamma+\alpha}}\left(\eta-\frac{\gamma+\alpha}{2 \gamma} \tau\right), \quad \phi=O\left(\tau^{2} H\right) \quad \text { when } \quad 1 \ll \tau \ll H^{-1 / 2} \\
\theta, \phi \sim \frac{1}{2} \operatorname{erfc}(\eta-\tau / 2) \quad \text { when } \quad H^{-1 / 2} \ll \tau .
\end{gathered}
$$

All these expressions arise in a quite straightforward manner either from (2.9), or else, for the large- $\tau$ cases, from the solution of (2.7), which corresponds to the single energy equation model for LTE. 
We note that, when $H \gg 1$, thermal equilibrium between the phases is achieved well before the effects of infiltration are felt but the converse is true when $H \ll 1$. We also remark that when $H=O(1)$ there is only one main transition close to unit values of $\tau$; the effects of the infiltration and the thermal coupling between the phases are felt simultaneously at that time, and there is no clearly identifiable intermediate regime.

If the above scenario were to prove to be the complete story then the problem would require little more analysis. However, we shall see in due course that the large- $\tau$ description is more complex than is suggested above and, indeed, unless $H$ is asymptotically large, the above simple-minded solution given in $(2.14 c)$ is erroneous. The analysis of Nouri-Borujerdi, Noghrehabadi \& Rees (2007) on the evolution of pure conduction after a sudden rise in boundary temperature shows that large values of $\tau^{2} H$ are equivalent to attaining LTE whatever the value of $H$, and then the evolving temperature profile is found to be independent of $H, \gamma$ and $\alpha$. In the present paper, which differs only by the presence of the infiltrating fluid, we shall discover that large values of $\tau^{2} H$ are also required for LTE between the phases, but the eventual thermal profile depends strongly on $H, \gamma$ and $\alpha$. This is a case where a naïve large- $\tau$ expansion like (2.14c) turns out to be misleading. The resolution of this paradox whereby a solution in LTE does not satisfy the single energy equation model (2.9) is given in $\S 4$.

\section{Numerical method}

Equations (2.9) subject to (2.11) were solved using a standard Keller-box scheme; see Keller (1978). The method is most often used for solving non-similar boundary layer problems, although it may be adapted easily to solving a variety of marching problems. Generally, the approach requires a reduction of the system to first-order form in $\eta$, which yields a parabolic system of four equations for the present simulations. Central differencing in both $\eta$ and $\tau$ yields a linear matrix/vector system for the unknowns at each time step. The matrix takes block tridiagonal form, and it is solved easily using the block-Thomas algorithm. At $\tau=0$ system (2.9) reduces to an ordinary differential form which may be solved using precisely the same central differencing technique in the $\eta$-direction.

Generally we took the step lengths in both directions to be 0.05 with the number of time steps taken depending on the maximum value of $\tau$ being considered. On the other hand, the spatial extent of the initial profile depends strongly on the chosen values of $H, \gamma$ and $\alpha$, as may be seen by the small- $\tau$ solutions presented in (2.12). Thus, if the function erfe $\eta$ is considered to have a boundary layer thickness equal to roughly 2, then the two main components of the solutions given in (2.12), namely $\operatorname{erfc} \zeta$ and erfc $\sqrt{\alpha} \zeta$, have boundary layer thicknesses which can be calculated easily. Account always had to be taken of how many grid points were necessary (i) to resolve the initial profiles adequately and (ii) to decide how large $\eta_{\max }$ should be at the beginning of the computation. Given that this is an infiltration problem, we also adjusted the value of $\eta_{\max }$ as the computation proceeded in order to account for the movement of the thermal front into the porous medium. Thus efficient use was made of smaller numbers of grid points at the beginning of the simulations without loss of accuracy.

There are three non-dimensional parameters at our disposal and, in order to assess the influence of each in a concise manner, we explore the effect of varying $H, \gamma$ and $\alpha$ in turn away from a chosen datum case in which $H=\gamma=\alpha=1$. Figures 1-3 show 

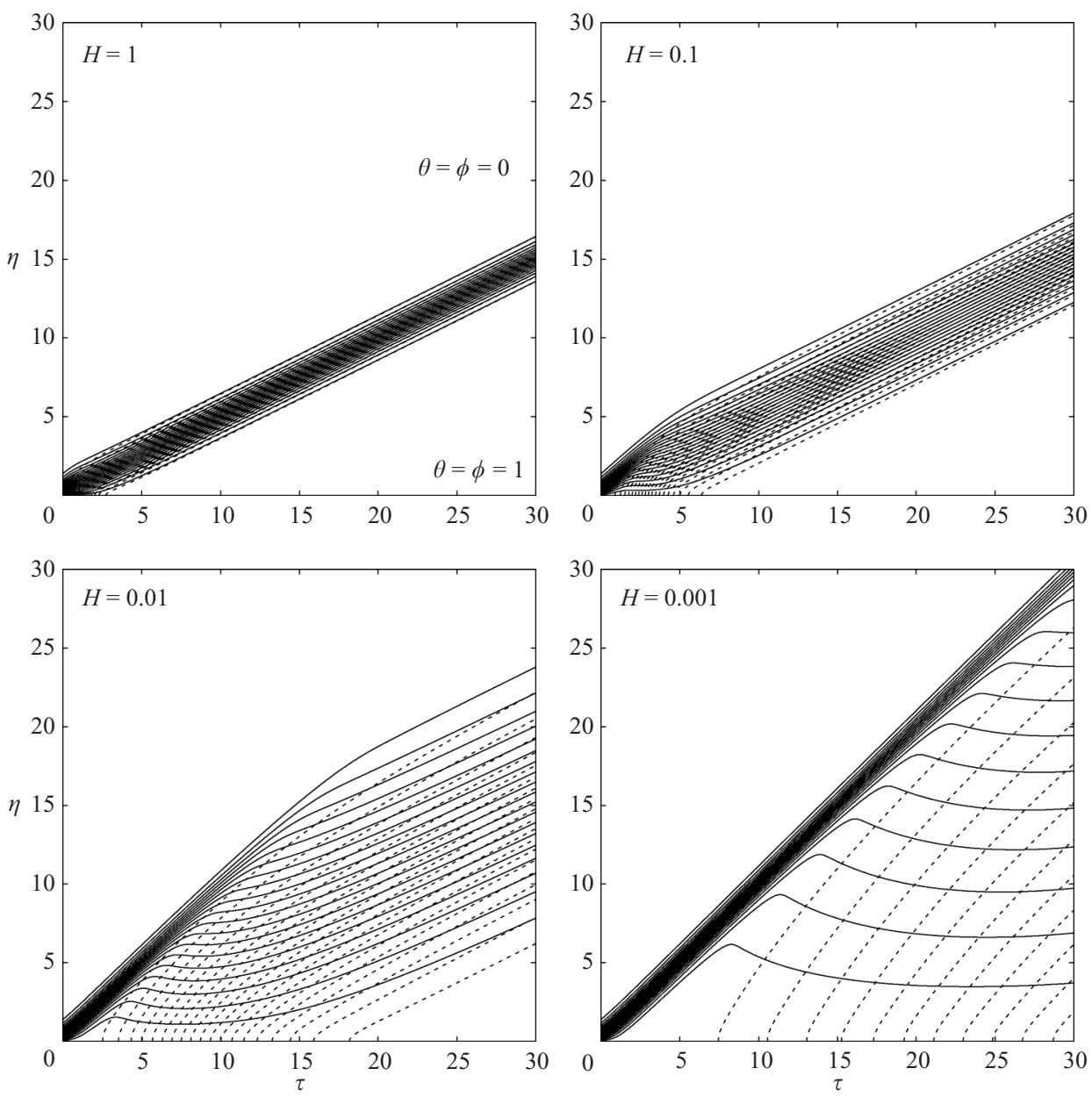

Figure 1. Contours of temperature of the fluid phase $\theta$ (continuous lines) and the solid phase $\phi$ (dashed lines). $\alpha=1$ and $\gamma=1$ for different values of $H$. The contours plotted correspond to temperature fields $0.05 n(n=0,1, \ldots, 20)$ in this and all other figures of the same type.

contours in $\eta-\tau$ space of the temperature fields of both phases. (We have chosen to use these variables for plotting purposes as the thickness of the thermal front tends to a constant as time progresses, and it is therefore easier to compare different solutions than if they were plotted in $x-t$ space.)

Figure 1 displays the effects of varying $H$, the inter-phase heat transfer coefficient, when $\gamma=\alpha=1$. Focusing first on the datum case, for which $H=1$, the thermal field of the fluid enlarges initially due to the fluid infiltration, and then a front of uniform width detaches from $\eta=0$ which propagates in the positive- $\eta$ direction, eventually without change of profile. As regards the thermal field of the solid phase, it is initially set at $\phi=0$, but evolves rapidly until its profile is almost indistinguishable from that of the fluid. In terms of the physical variables, $x$ and $t$, the fully developed front is indeed travelling at a uniform velocity: at sufficiently large values of $\tau$, the $\theta=0.5$ contour lies along $\eta=\tau / 2$ almost precisely, and this is equivalent to $x=t$. Therefore the front is in a state of LTE and is travelling with the speed of the infiltrating fluid, although it is also expanding slowly in the $x$-direction owing to diffusion. 
As $H$ takes increasingly large values, the thermal profile of the solid phase adjusts increasingly rapidly to that of the fluid phase due to the increased importance of the source/sink terms in (2.9). On the other hand, when $H$ decreases below unity, not only is there an lengthening time delay before the phases become close to LTE, but the width of the front increases. Thus we have our first evidence that the large- $\tau$ behaviour proposed in $(2.14 c)$ using a simple-minded approach is actually incorrect, for that expression is independent of $H$. Indeed when $H$ takes very small values, such as $H=0.001$ (the last panel in figure 1) then once the thermal front of the fluid has detached itself from the boundary, the evolving front satisfies $(2.14 b)$, which, given that $\alpha=\gamma=1$ here, becomes $\theta \sim(1 / 2) \operatorname{erfc}(\eta-\tau)$. Therefore the centre of the front is located at $x=2 t$, and it is clearly travelling faster than when the fluid and solid phases are in LTE. In particular, this means that the front has non-dimensional velocity equal to 2 , which is twice the velocity of the infiltrating fluid. Although this conclusion appears to be counter-intuitive, it is important to realize that fluid velocities in porous media are superficial averages, i.e. they are averages taken over both phases. The intrinsic average velocity of the fluid is higher, as this average is taken over the fluid phase only. More generally, then, at intermediate times, the thermal front is given by $(2.14 b)$, and the centre of this front is at $x^{*}=U t^{*} / \varepsilon$ after conversion back into dimensional form using (2.2) and the definition of $U$. Therefore the dimensional velocity of the front is $U / \varepsilon$, which is precisely the intrinsic average of the velocity of the infiltrating fluid.

Figure 2 shows the effects of different values of $\gamma$ on the evolution of the thermal fronts when $H=\alpha=1$. There is a great deal of qualitative similarity between these cases and those given in figure 1. The primary reason is that $\gamma$ appears in the source/sink term in equation $(2.9 b)$ for the solid phase. Therefore large values serve to enhance LTE while small values tend to decouple the thermal energy equations, thereby favouring LTNE. Notice that the presence of $\gamma$ in other coefficients causes some quantitative details to vary but these dependences do not affect the qualitative behaviours of solutions. The velocity of the front for the fluid phase increases with decreasing values of $\gamma$, whereas figure 1 shows that it is independent of $H$ when $H$ decreases. The fluid-phase isotherms for $\gamma=0.1$ appear to indicate that the front reverses its direction, but this is simply an artifact of the $\eta-\tau$ coordinate system used; all isotherms, when plotted in terms of $x$ and $t$, have a positive slope.

Figure 3 shows the corresponding isotherms for different values of $\alpha$ while $H=\gamma=1$. As the parameter $\alpha$ is a diffusivity ratio, large values correspond to a more highly conducting fluid phase. In addition, from (2.13a) and $(2.14 a)$, large values of $\alpha$ also give rise to a relatively thick thermal boundary layer in the fluid phase; this is seen quite clearly in figure 3 for the cases $\alpha=100$ and $\alpha=10$. When $\alpha$ takes small values, there is preferential conduction in the solid phase, which is seen clearly for the cases where $\alpha<1$. In these latter situations, the small size of $\alpha$, which multiplies the $\phi_{\tau}$ term in $(2.9 b)$ and which therefore enhances the effect of the source/sink term in that equation, serves to heat up the solid phase very rapidly. The relative ease of conduction allows the thermal field to spread out ahead of that of the fluid phase despite the fact that the fluid is moving.

There are two aspects of the solutions presented so far which are worthy of further exploration, namely, the large-time and the small- $H$ behaviours of the system. The former issue is of interest because it is clear that the evolving front tends towards a state of uniform thickness and the extent of the front depends on the non-dimensional parameters. That this is so is surprising, for it was anticipated that the increasing magnitude of the coefficients of the source/sink terms would 

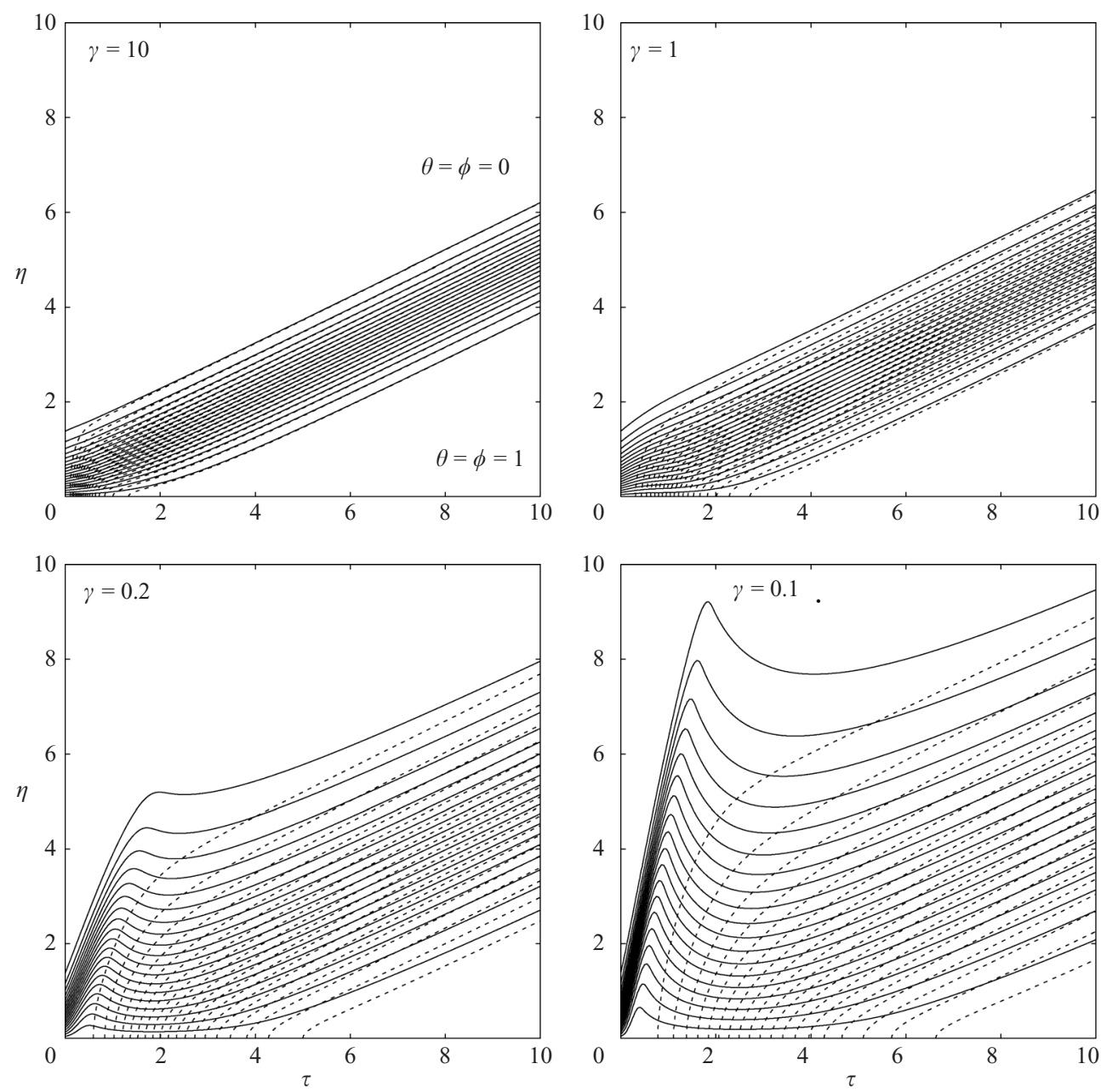

Figure 2. Contours of temperature of the fluid phase (continuous lines) and the solid phase (dashed lines). $H=1$ and $\alpha=1$ for different values of $\gamma$.

guarantee that LTE would be approached for any set of values of $H, \gamma$ and $\alpha$, and therefore the large- $\tau$ solution should be given by $(2.13 c)$ and $(2.14 c)$ independent of the particular parameter values at hand. As regards the small- $H$ structure, the approach to a travelling wave solution slows markedly as $H$ decreases towards zero, and the resulting front is very wide. Therefore the diffusion terms in (2.9) are likely to play largely passive roles once the thermal front of the fluid phase has detached itself from the boundary. When $H \ll 1$ the governing equations change from being parabolic to being hyperbolic in nature.

\section{Large-time asymptotic solution}

The numerical solutions summarized in figures 1-3 suggest that the temperature profiles of the two phases tend to one another as $\tau \rightarrow \infty$. The aim here is to sketch an asymptotic analysis which confirms this observation and, moreover, explains how the thickness of the thermal front depends on the governing parameters. 

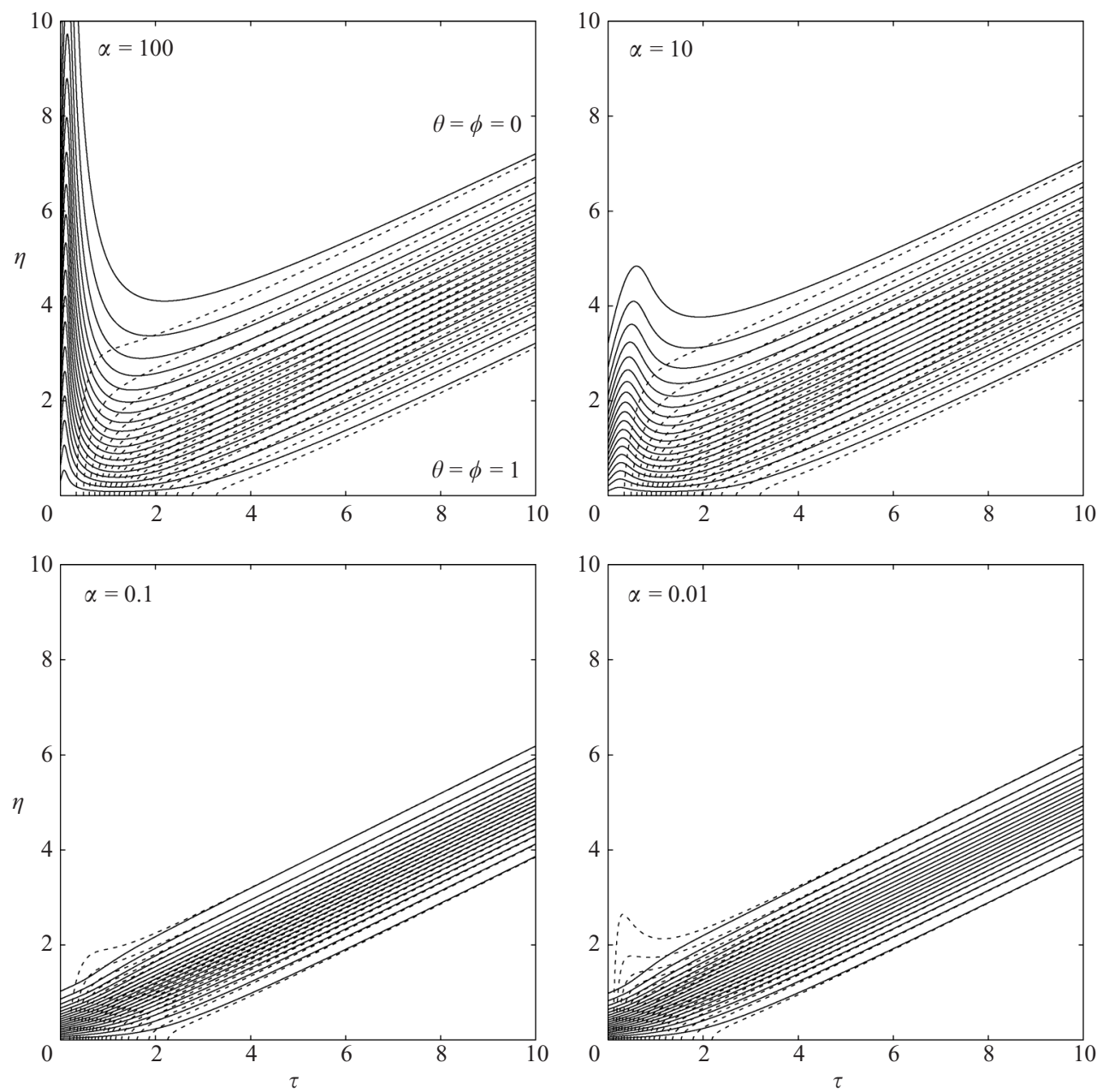

FIGURE 3. Contours of temperature of the fluid phase (continuous lines) and the solid phase (dashed lines). $H=1$ and $\gamma=1$ for different values of $\alpha$.

The numerical solutions indicate clearly the importance of the line of solution $\eta=\tau / 2$, so let us redefine the spatial coordinate

$$
\eta=\frac{1}{2} \tau+\zeta
$$

then $\zeta=0$ corresponds to the line of apparent activity in the centre of the front. Written in terms of $\tau$ and $\zeta$, system (2.9) becomes

$$
\begin{aligned}
& \gamma\left(\frac{\gamma+1}{\gamma+\alpha}\right) 2 \tau \frac{\partial \theta}{\partial \tau}=\gamma \frac{\partial^{2} \theta}{\partial \zeta^{2}}+2\left(\frac{\gamma+1}{\gamma+\alpha}\right)[\gamma \zeta-\alpha \tau] \frac{\partial \theta}{\partial \zeta}+4 \tau^{2} H \gamma(\phi-\theta) \\
& \alpha\left(\frac{\gamma+1}{\gamma+\alpha}\right) 2 \tau \frac{\partial \phi}{\partial \tau}=\frac{\partial^{2} \phi}{\partial \zeta^{2}}+2\left(\frac{\gamma+1}{\gamma+\alpha}\right)[\alpha \zeta+\alpha \tau] \frac{\partial \phi}{\partial \zeta}+4 \tau^{2} H \gamma(\theta-\phi)
\end{aligned}
$$

where these new equations have been arranged to display as much symmetry as possible. The boundary conditions require that $\theta=1$ and $\phi_{\zeta}=0$ at $\zeta=-\tau / 2$, and that both $\theta$ and $\phi$ vanish as $\zeta \rightarrow \infty$. 
We seek a solution of the form

$$
(\theta, \phi)=\left(\theta_{0}, \phi_{0}\right)+\frac{1}{\tau}\left(\theta_{1}, \phi_{1}\right)+\frac{1}{\tau^{2}}\left(\theta_{2}, \phi_{2}\right)+\cdots,
$$

with each $\theta_{j}$ and $\phi_{j}$ functions of $\zeta$. We substitute into (4.2) and compare like powers of $\tau$. At $O\left(\tau^{2}\right)$ we just obtain the consistency condition $\phi_{0}=\theta_{0}$. The two balances that arise from $(4.2 a, b)$ at $O(\tau)$ combine to show that $\phi_{1}$ and $\theta_{1}$ are related by

$$
\phi_{1}=\theta_{1}+\frac{\alpha}{2 \gamma H}\left(\frac{\gamma+1}{\gamma+\alpha}\right) \frac{\mathrm{d} \theta_{0}}{\mathrm{~d} \zeta}
$$

irrespective of the precise form of the zeroth-order unknowns. It is not until the next order that these quantities are tied down, for $O(1)$ terms in $(4.2 a, b)$ tell us that

$$
\begin{aligned}
& \gamma \frac{\mathrm{d}^{2} \theta_{0}}{\mathrm{~d} \zeta^{2}}+\gamma\left(\frac{\gamma+1}{\gamma+\alpha}\right) 2 \zeta \frac{\mathrm{d} \theta}{\mathrm{d} \zeta}-2 \alpha\left(\frac{\gamma+1}{\gamma+\alpha}\right) \frac{\mathrm{d} \theta_{1}}{\mathrm{~d} \zeta}+4 H \gamma\left(\phi_{2}-\theta_{2}\right)=0, \\
& \alpha \frac{\mathrm{d}^{2} \phi_{0}}{\mathrm{~d} \zeta^{2}}+\alpha\left(\frac{\gamma+1}{\gamma+\alpha}\right) 2 \zeta \frac{\mathrm{d} \phi}{\mathrm{d} \zeta}+2 \alpha\left(\frac{\gamma+1}{\gamma+\alpha}\right) \frac{\mathrm{d} \phi_{1}}{\mathrm{~d} \zeta}+4 H \gamma\left(\theta_{2}-\phi_{2}\right)=0 .
\end{aligned}
$$

If we add these two equations and use the previous results, we have that $\theta_{0}$ satisfies

$$
\left[1+\frac{\alpha^{2}(\gamma+1)}{H \gamma(\gamma+\alpha)^{2}}\right] \frac{\mathrm{d}^{2} \theta_{0}}{\mathrm{~d} \zeta^{2}}+2 \zeta \frac{\mathrm{d} \theta_{0}}{\mathrm{~d} \zeta}=0
$$

and in view of the boundary conditions the appropriate solution of (4.6) is

$$
\theta_{0}=\frac{1}{2} \operatorname{erfc}(Z)
$$

where

$$
Z \equiv\left[1+\frac{\alpha^{2}(\gamma+1)}{H \gamma(\gamma+\alpha)^{2}}\right]^{-1 / 2} \zeta
$$

Strictly this solution contains superexponentially small errors when $\tau$ is large since the boundary is at $\zeta=-\tau / 2$ (rather than as $\zeta \rightarrow-\infty$ ), but our purposes do not require consideration of such tiny terms. If, to be precise, we define the front to be that part lying in the range $-1 \leqslant Z \leqslant 1$, then its thickness in terms of $\zeta$ is

$$
\zeta_{\text {front }}=2\left[1+\frac{\alpha^{2}(\gamma+1)}{H \gamma(\gamma+\alpha)^{2}}\right]^{1 / 2} .
$$

It is clear from this expression that the evolving front yields the expected LTE thickness, namely 2, given our present definition, in the separate limits, $H \rightarrow \infty$, $\gamma \rightarrow \infty$ and $\alpha \rightarrow 0$. On the other hand, the front becomes very thick in the separate limits $H \rightarrow 0$ or $\gamma \rightarrow 0$.

Figure 4 compares the leading-order large- $\tau$ asymptotic profiles given by equation (4.6) with those obtained by using the Keller-box code. The parameters $H=0.1$ and $\gamma=\alpha=1$ were chosen, and the profiles were compared for four different values of $\tau$. For this parameter set the asymptotic solution yields quite an accurate representation of the thermal profile of each phase since the phases are close to being in LTE. The figure also gives some indication of the rapidity with which the numerical solutions approach the asymptotic solution. The solutions presented in figure 1 indicate that the time beyond which the asymptotic solution is valid depends strongly on the precise values of the non-dimensional parameters. Equation (4.4) gives the leading-order difference between the temperature profiles of the phases, which is 


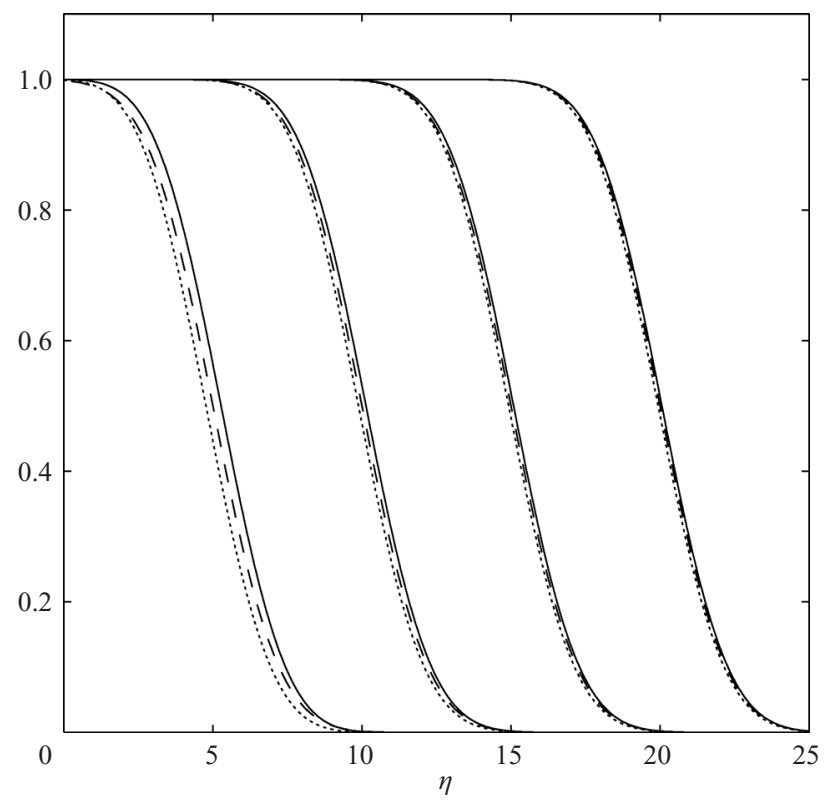

Figure 4. Comparison of the numerically computed profiles for $\theta$ (solid lines) and $\phi$ (dotted lines) with the leading-order large- $\tau$ asymptotic solution (long dashes) given by (4.7). The parameter values are $H=0.1, \gamma=\alpha=1$, while $\tau=10,20,30$ and 40.

the thermal wave described in Kuznetsov (1994). Given the form of $\theta_{0}$ in (4.7), this difference is maximized at $\zeta=Z=0$. Therefore we find that the maximum difference in the temperatures takes the form

$$
\theta-\phi \sim \frac{\alpha}{\sqrt{\pi} H \gamma}\left(\frac{\gamma+1}{\gamma+\alpha}\right)\left(1+\frac{\alpha^{2}(\gamma+1)}{H \gamma(\gamma+\alpha)^{2}}\right)^{-1 / 2} \tau^{-1}
$$

where it is noted that $\theta>\phi$ in this regime. Given the manner of the time-dependence of this quantity (proportional to $t^{-1 / 2}$ ), we see how the rapidity of the approach to LTE (or thermalization; see Abu-Hijleh et al. 2004) depends on the governing parameters. In particular when $H$ takes very large or very small values, we have

$$
\theta-\phi \sim \frac{\alpha}{2 \sqrt{\pi} H \gamma}\left(\frac{\gamma+1}{\gamma+\alpha}\right) \tau^{-1}, \quad \text { or } \quad \theta-\phi \sim \frac{1}{2} \sqrt{\frac{\gamma+1}{\pi H \gamma}} \tau^{-1},
$$

respectively. For large values of $H$ LTE is achieved very rapidly, but the time for the establishment of LTE increases greatly as $H$ decreases and we now concentrate on further description of this case.

\section{Small- $H$ analysis: the hyperbolic approximation}

In order to investigate the behaviour of the system in the small- $H$ limit, it is convenient to rescale (2.4) once more; setting

$$
\bar{t}=H \frac{\gamma+\alpha}{\gamma+1} t, \quad \bar{x}=H \frac{\gamma}{\gamma+1} x
$$


leads to the system

$$
\begin{aligned}
\frac{\partial \theta}{\partial \bar{t}}+\frac{\partial \theta}{\partial \bar{x}} & =H\left(\frac{\gamma}{\gamma+1}\right)^{2} \frac{\partial^{2} \theta}{\partial \bar{x}^{2}}+(\phi-\theta), \\
\alpha \frac{\partial \phi}{\partial \bar{t}} & =H\left(\frac{\gamma}{\gamma+1}\right)^{2} \frac{\partial^{2} \phi}{\partial \bar{x}^{2}}+\gamma(\theta-\phi) .
\end{aligned}
$$

Therefore, when $\bar{H} \equiv H(\gamma /(\gamma+1))^{2} \ll 1$, the governing equations change type from parabolic to hyperbolic. Given that

$$
\bar{H}=\frac{h L^{2} \varepsilon k_{\mathrm{f}}}{k_{\mathrm{pm}}^{2}}=\frac{h \varepsilon k_{\mathrm{f}}}{U^{2}(\rho C)_{\mathrm{f}}^{2}},
$$

we see that the system may be regarded as a hyperbolic one when $U$ is sufficiently large, or conversely, when one or more of $h, k_{\mathrm{f}}$ or $\varepsilon$ are sufficiently small. In this case shocks are expected to occur which are equivalent to sharp changes in the temperature of the fluid phase and, possibly, of the solid phase as well.

Therefore the system we study here is deduced by taking $\bar{H} \rightarrow 0$, whereupon

$$
\theta_{\bar{t}}+\theta_{\bar{x}}=\phi-\theta, \quad \phi_{\bar{t}}=-c(\phi-\theta)
$$

where $c=\gamma / \alpha$ is a constant; the required boundary conditions are that $\theta=\phi=0$ initially and $\theta=1$ on $\bar{x}=0$. This system is similar in style to that considered by Chikwendu \& Ojiakor (1985) who studied a more complicated version including some diffusion terms. They demonstrated how transform methods could be used to solve their equations, although the necessary inversions could only be carried out in certain special cases; in general only asymptotic forms of the solution could be deduced. In contrast, our simpler equations (5.4) admit an exact solution which can be obtained using the Laplace transform. If we define

$$
\mathscr{L}[\theta]=\Theta(\bar{x}, p)=\int_{0}^{\infty} \theta(\bar{x}, \bar{t}) \exp (-p \bar{t}) \mathrm{d} \bar{t}
$$

and identify $\Phi(\bar{x}, p) \equiv \mathscr{L}[\phi]$, then transforms of system (5.4) give that

$$
\Theta=\frac{1}{p} \exp \left[\frac{c \bar{x}}{p+c}-\bar{x}(1+p)\right] .
$$

It is known (Gradshteyn \& Ryzhik 1980) that

$$
\mathscr{L}\left[\frac{I_{1}(2 \sqrt{\bar{t}})}{\sqrt{\bar{t}}}\right]=\exp \left(\frac{1}{p}-1\right),
$$

where $I_{n}$ denotes the usual modified Bessel function of order $n$. This result, when combined with the shift theorem for Laplace transforms and the convolution theorem, enables us to invert (5.6). Routine calculations yield

$$
\theta=\mathrm{e}^{-\bar{x}}\left[1+\int_{0}^{2 \sqrt{c \bar{x}(\bar{t}-\bar{x})}} \exp \left(-\frac{u^{2}}{4 \bar{x}}\right) I_{1}(u) \mathrm{d} u\right] \quad \text { for } \quad \bar{x} \leqslant \bar{t} ;
$$

on $\bar{x}=\bar{t}$ then $\theta=\mathrm{e}^{-\bar{x}}$. It then follows that

$$
\phi=\theta-\mathrm{e}^{c(\bar{x}-\bar{t})-\bar{x}} I_{0}(2 \sqrt{c \bar{x}(\bar{t}-\bar{x})}) .
$$

Although this establishes formally exact solutions for $\theta$ and $\phi$, it is not immediately clear how these behave in the $(\bar{x}, \bar{t})$ plane. In order to probe a little deeper, it is 
helpful to examine the asymptotics of the integral in (5.8) when $\bar{x}=\lambda \bar{t}$ with $\bar{t} \gg 1$ and the constant $\lambda<1$. If we make an obvious change of variables then

$$
M \equiv \int_{0}^{2} \sqrt{c \bar{x}(\bar{t}-\bar{x})} \exp \left(-\frac{u^{2}}{4 \bar{x}}\right) I_{1}(u) \mathrm{d} u=\bar{t} \sqrt{\lambda} \int_{0}^{2 \sqrt{c(1-\lambda)}} \exp \left(-\frac{\bar{t} w^{2}}{4}\right) I_{1}(\bar{t} w \sqrt{\lambda}) \mathrm{d} w
$$

and we expect the dominant contribution to the integral to arise from the vicinity of the maximum point of the integrand. Standard results (Abramowitz \& Stegun 1965) give the form of the exponential growth of $I_{1}$ with increasing argument, and a quick inspection of (5.10) suggests that if we use the leading term for $I_{1}$ then

$$
M \sim \frac{\bar{t}^{1 / 2} \lambda^{1 / 4}}{\sqrt{2 \pi}} \mathrm{e}^{\bar{t} \lambda} \int_{0}^{2 \sqrt{c(1-\lambda)}} w^{-i-1 / 2} \exp \left(-\frac{\bar{t}}{4}(w-2 \sqrt{\lambda})^{2}\right) \mathrm{d} w .
$$

The conclusion is that the largest contribution to $M$ ought to arise in the vicinity of $w=2 \sqrt{\lambda}$, as long as, of course, this point lies within the range of integration; otherwise the integrand is largest at the right-hand end of the integration range. It is an easy exercise to compute the asymptotic behaviour of $M$ for $\bar{t} \gg 1$ but there are then two regimes that may occur, depending on whether or not $2 \sqrt{\lambda}<2 \sqrt{c(1-\lambda)}$.

After some lengthy evaluations it is found that, recalling that $\bar{x}=\lambda \bar{t}, \lambda<1$ and $\bar{t} \gg 1:$ if $\lambda<c /(1+c)$ then

$$
\theta=1+o\left(\bar{t}^{-1}\right) \text { and } \phi \sim \theta .
$$

In fact we can be more precise as to the relationship between $\theta$ and $\phi$ in this regime, for (5.9) reveals that these fields differ by only an exponentially small quantity.

On the other hand, when $\lambda>c /(1+c)$ it follows that

$$
\begin{aligned}
& \theta \sim \frac{1}{2 \sqrt{\pi \bar{t}}}\left(\frac{\lambda}{c(1-\lambda)}\right)^{1 / 4} \frac{\sqrt{\lambda}+\sqrt{c(1-\lambda)}}{(1+c) \lambda-c} \exp [\bar{t}[2 \sqrt{c \lambda(1-\lambda)}+\lambda(c-1)-c]] \\
& \phi \sim \frac{1}{2 \sqrt{\pi \bar{t}}}\left(\frac{c(1-\lambda)}{\lambda}\right)^{1 / 4} \frac{\sqrt{\lambda}+\sqrt{c(1-\lambda)}}{(1+c) \lambda-c} \exp [\bar{t}[2 \sqrt{c \lambda(1-\lambda)}+\lambda(c-1)-c]] .
\end{aligned}
$$

We point out that the apparent discontinuities in solutions (5.12) and (5.13) can easily be resolved by a careful consideration of the behaviour of the integral $M$ for $\lambda$ in the vicinity of $\lambda=c /(1+c)$. Indeed, for $\lambda=c /(1+c)+\bar{t}^{-1 / 2} \Delta$, with $\Delta=O(1)$, it may be shown that

$$
\theta \sim \frac{1}{2 \sqrt{\pi}} \int_{-\infty}^{-\Delta \sqrt{(1+c)^{3} / c}} \exp \left(-\frac{1}{4} S^{2}\right) \mathrm{d} S
$$

and it is then a routine task to show that this form matches smoothly onto (5.12) and $(5.13 a)$ as $\Delta \rightarrow \pm \infty$.

Figures 5 and 6 show some solutions obtained using Laplace transforms compared with selected parabolic solutions for small values of $H$. Figure 5 illustrates contours of the temperatures for both phases for the case $c=1$, where $\gamma=\alpha=1$ has been taken for the parabolic simulations. These contours have been displayed in $\bar{x}-\bar{t}$ space for the Laplace transform solutions but in $x-t$ space for the parabolic simulations. However, the ranges of values chosen for the two cases correspond precisely given the selected values of $H, \gamma$ and $\alpha$. 

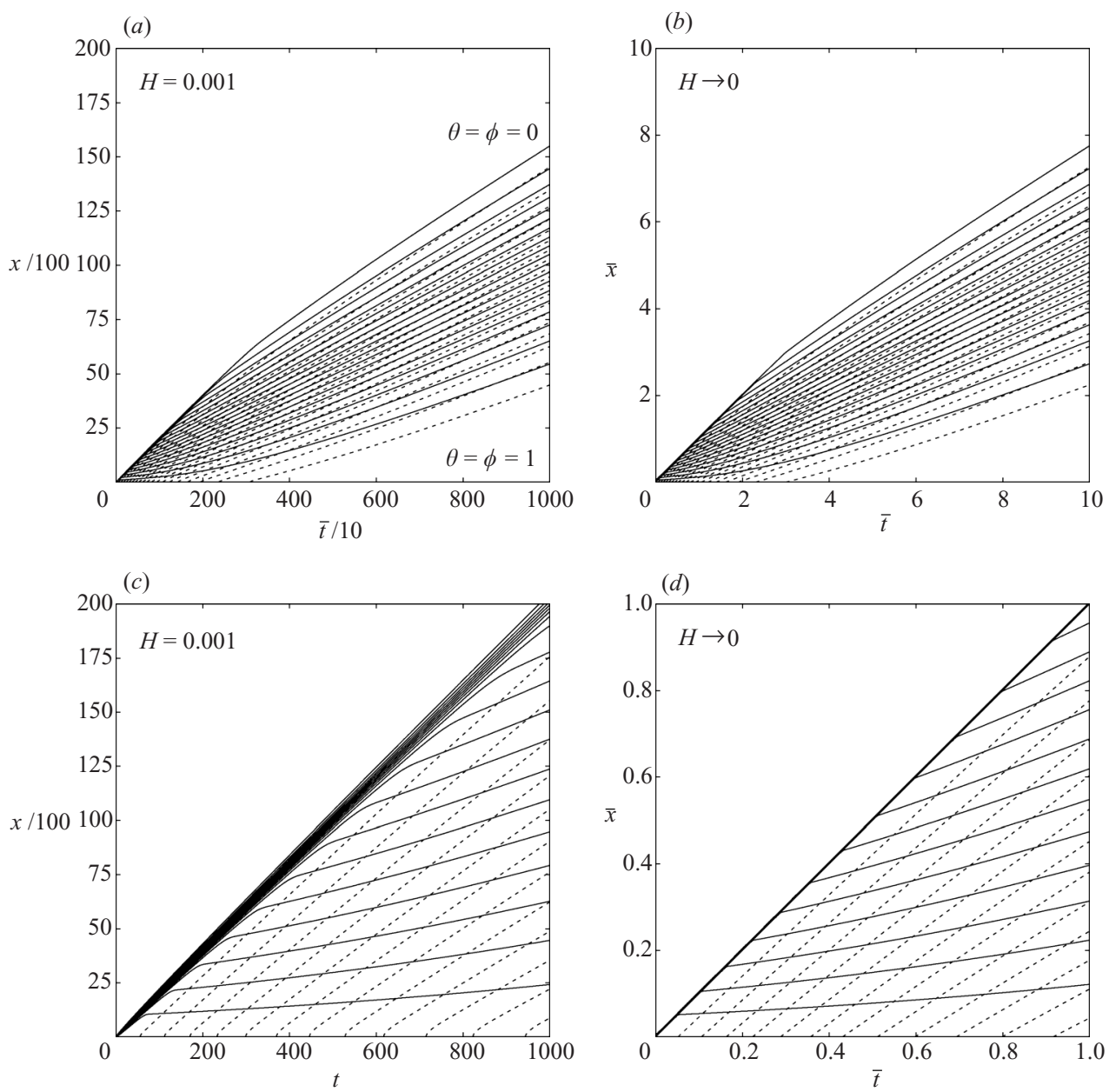

FIGURE 5. Comparison between the parabolic simulations for $H=0.001(a, c)$, and the approximate hyperbolic solutions $(b, d)$ displaying isotherms for the fluid phase (continuous lines) and the solid phase (dashed lines). We have taken $c=1$ with $\gamma=\alpha=1$.

Figures $5(b)$ and $5(d)$ correspond to the Laplace transform solution of the hyperbolic equations. A thermal shock wave is evidently set up close to $\bar{x}=\bar{t}=0$. As time progresses so the strength of the shock appears to reduce and the integral given in (5.8) suggests that the height of the shock is precisely exp $(-t)$. Therefore the shock degrades as time progresses, due to the fact that the fluid phase surrenders heat continually, though slowly, to the solid phase. Figure $5(a)$ and $5(b)$ clearly compare exceptionally well, with the only noticeable difference being the presence of a distinct corner in the $\theta=0.05$ isotherm at $\bar{t}=\bar{x}=3$.

Figures $5(c)$ and $5(d)$ display the thermal fields much closer to $\bar{x}=\bar{t}=0$. In this range there is a very distinct contrast between the parabolic and the hyperbolic simulations. A small amount of diffusion is evident in the parabolic simulations which serves to smooth the thermal shock wave. Apart from the fluid-phase isotherms in the shock region, the corresponding isotherms of each phase compare extremely well, showing that diffusion is generally unimportant. Therefore, when $H \ll 1$, the transform solution 

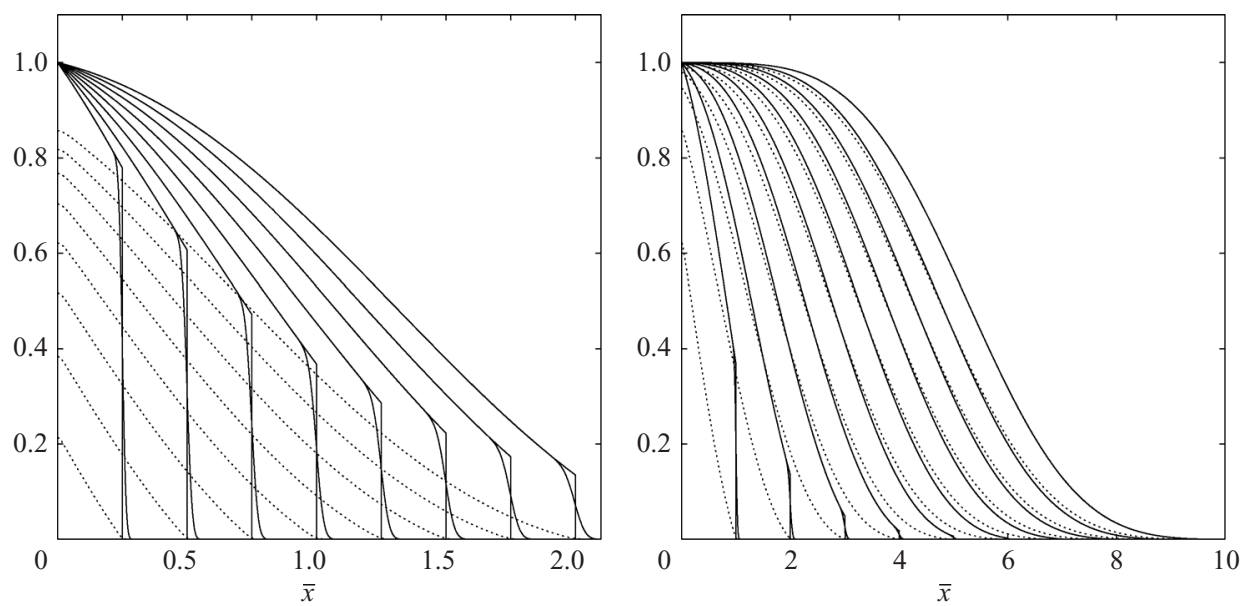

Figure 6. Profiles of temperature of the fluid phase (continuous lines) and the solid phase (dashed lines) in the small- $H$ limit. We have taken $\alpha=1$ and $\gamma=1$ with $c=1$. For the fluid phase the hyperbolic $H \rightarrow 0$ solutions display shocks, while the smooth curves correspond to $H=0.001$. For the solid phase the $H \rightarrow 0$ and $H=0.001$ curves are indistinguishable.

may be used to estimate thermal breakthrough time without the need to resort to detailed numerical calculations.

Figure 6 shows how the detailed temperature profiles behave in time for the parameter set corresponding to figure 5 , and it also gives a comparison between the parabolic and hyperbolic solutions. In general, there is no discernible difference between the two different solutions for the solid phase, and almost no difference in the profiles for the fluid phase either. The only exception occurs at the shock front, and this figure shows very clearly the increasing effect of diffusion as the shock weakens with time.

Both figures 5 and 6 suggest that there remains quite a substantial difference between the thermal profiles of the two phases, with the solid phase still lagging behind the fluid phase at $\bar{t}=10$. When $c=1$, which is the value chosen here, we may use equation (5.9) to determine the maximum value of $\theta-\phi$ at any given time. On assuming that the argument in the function $I_{0}$ is large, we may use its leading-order asymptotic form to deduce that

$$
\theta-\phi \sim \frac{\mathrm{e}^{-\bar{x}+2} \sqrt{\bar{x}(\bar{t}-\bar{x})}}{2 \sqrt{\pi}[\bar{x}(\bar{t}-\bar{x})]^{1 / 4}} .
$$

For $\bar{t} \gg 1$ this function is largest very close to $\bar{x}=\mu \bar{t}$ say, where $\mu$ is chosen so as to maximize the exponent in the numerator. This exponent is

$$
E=[2 \sqrt{\mu(1-\mu)}-1] \bar{t},
$$

which is greatest for $\mu=1 / 2$, whence $E=0$. Then equation (5.15) reduces to

$$
\theta-\phi \sim \frac{1}{\sqrt{2 \pi t}}
$$


which implies that LTE is attained very slowly. The equivalent expression for general values of $c$ is

$$
\theta-\phi \sim \sqrt{\frac{1+c}{c}} \frac{1}{2 \sqrt{\pi t}},
$$

which, when translated back into the original non-dimensional variables, becomes

$$
\theta-\phi \sim \frac{1}{2} \sqrt{\frac{\gamma+1}{\pi H \gamma}} t^{-1 / 2},
$$

and is precisely the same as that given in equation (4.11) for small values of $H$. Therefore it appears that the $H \rightarrow 0$ and $t \rightarrow \infty$ limits yield the same conclusion irrespective of the order in which the limits are taken. This is somewhat surprising because the large-time parabolic analysis of $\S 4$ has diffusion playing a central role, even when $H$ takes asymptotically small values, whereas the small- $H$ analysis of the present section is one in which diffusion is negligible.

Finally, if for the sake of estimating time scales we were to define LTE as being attained $\theta-\phi<0.01$ for all values of $x$ at large times, then LTE occurs when

$$
t>\frac{10^{4}(\gamma+1)}{4 \pi H \gamma} \text {. }
$$

\section{Discussion and conclusions}

In this paper we considered in detail the manner in which the temperature fields of the solid and fluid phases evolve when a hot fluid is suddenly injected into a cold saturated porous medium. In particular we have concentrated on those cases where the phases are not in LTE. In using the simplest possible two-equation model to describe the evolution of the temperature fields of each phase, we have employed fully numerical means, a large-time analysis and a strong LTNE hyperbolic analysis to understand the variety of phenomena this apparently simple problem is able to display, even in a one-dimensional idealized system.

We have shown that LTE is always attained as $t \rightarrow \infty$. However, once LTE has been reached, the evolving thermal front is not the one that would be obtained had LTE been assumed at the outset. Rather, the early, very strong LTNE effects and the associated slow response of the solid phase to the changing thermal profile of the fluid phase gives rise to a thermal front with a width which depends on the values of the three governing parameters. Thus a thicker front is formed than the one obtained when $H$ is asymptotically large. We believe that this is the first time that a situation has been described where a solution in LTE still depends on the values of $H, \gamma$ and $\alpha$ - this is a reason why the naïve large- $\tau$ solution given in $(2.14 c)$ seemed reasonable a priori. By contrast, the thermal front studied by Nouri-Borujerdi et al. (2007), which also eventually attains LTE irrespective of the values of the governing parameters, gives rise to an evolving front which is independent of the governing non-dimensional parameters. The steady boundary layer analysis of Rees (2003) also yields a parameter-independent flow at sufficiently large distances from the leading edge.

While the solid phase is slow to react initially to the advancing hot fluid when the phases are in strong LTNE, there are situations where the solid phase responds very quickly. In particular, very small values of $\alpha$ correspond to a relatively high thermal diffusivity of the solid phase, a situation which is unlikely to arise in geothermal 
contexts. But in these situations, which could model heat exchangers where the solid phase is metallic, conduction in the solid phase will advance rapidly at early times, overtaking the combined effects of conduction and advection in the fluid phase. It comes, therefore, as little surprise that the asymptotic width of the evolving front is then very close to that obtained when the phases are in LTE, since the speed of the fluid will eventually become substantially larger than the thermal diffusion speed, which is proportional to $t^{-1 / 2}$. This physical conclusion is backed by the expression given in equation (4.9) for the width of the advancing thermal front. Small values of $\alpha$ contribute little change to the width of the front.

At sufficiently high flow rates, diffusion may be neglected formally, in line with the studies of Shook (2001) and Stopa \& Wojnarowski (2006). Therefore a thermal shock wave in the fluid phase is obtained whose strength decays in time. This result is also qualitatively different from the LTE situation, as exemplified by equation (2.7), in which, when diffusion has been neglected, a shock wave of constant height is obtained. Therefore, the presence of heat transfer between the phases causes the strength of the thermal shock to be degraded with time.

The situation we have considered in this paper is perhaps the simplest in which LTNE effects might be considered. Referring to the geothermal applications, both injection and production wells are line sources and sinks, and the resulting flow field is either two- or, more typically, three-dimensional. In addition, we have assumed that buoyancy forces can be neglected and these too will add to the complexity of the flow field. However, our intention here has been to uncover how the presence of LTNE alters a relatively straightforward basic case, and our conclusion is that substantial changes do indeed take place when LTNE effects are strong. It is anticipated that similar alterations will also occur within more complex configurations.

P. G. S. would like to acknowledge the receipt of a Royal Society Incoming Fellowship, to thank the University of Bath for its hospitality and to thank Bangalore University for granting him leave. The authors would like to thank the anonymous referees for their helpful suggestions.

\section{REFERENCES}

Abramowitz, M. \& Stegun, I. A. 1965 Handbook of Mathematical Functions. Dover.

Abu-Hijleh, B. A., Al-Nimr, M. A. \& Hader, M. A. 2004 Thermal equilibrium in transient forced convection porous channel flow. Transp. Porous Media 57, 49-58.

AnZelius, A. 1926 Über Erwärmung vermittels durchströmender Medien. Zeit. Ang. Math. Mech. 6, 291-294.

Banu, N. \& Rees, D. A. S. 2001 Onset of Darcy-Bénard convection using a thermal nonequilibrium model. Intl J. Heat Mass Transfer 45, 2221-2228.

BARBIER, E. 2002 Geothermal energy technology and current status: An overview. Renewable \& Sustainable Energy Reviews 6, 3-65.

BAYTAS, A. C. 2003 Thermal non-equilibrium natural convection in a square enclosure filled with a heat-generating solid phase, non-Darcy porous medium. Intl J. Energy Res. 27, 975-988.

Baytas, A. C. \& Pop, I. 2002 Free convection in a square porous cavity using a thermal nonequilibrium model. Intl J. Thermal Sci. 41, 861-870.

Bodvarsson, G. 1972 Thermal problems in the siting of reinjection wells. Geothermics 1, 63-66.

Burch, D. M., Allen, R. W. \& Peavy, B. A. 1976 Transient temperature distributions within porous slabs subject to sudden transpiration heating. Trans. ASME J. Heat Transfer 98, 221-225.

Carslaw, H. S. \& Jaeger, J. C. 1959 Conduction of Heat in Solids. Oxford University Press.

Chikwendu, S. C. \& OJiakor, G. U. 1985 Slow-zone model for longitudinal dispersion in twodimensional shear flows. J. Fluid Mech. 152, 15-38. 
Combarnous, M. \& Bories, S. A. 1974 Modélisation de la convection naturelle au sein d'une couche poreuse horizontal à l'aide d'un coefficient de transfert solide-fluide. Intl J. Heat Mass Transfer 17, 505-515.

Ennis-King, J., Preston, I. \& Paterson, L. 2005 Onset of convection in anisotropic porous media subject to a rapid change in boundary conditions. Phys. Fluids 17, 084107-1-084107-15.

Gradshteyn, I. S. \& RyzhiK, I. M. 1980 Table of Integrals, Series and Products, 4th edition. Academic Press.

Keller, H. B. 1978 Numerical methods in boundary layer theory. Annu. Rev. Fluid Mech. 10, 417-433.

KuzNETSOv, A. V. 1994 An investigation of a wave of temperature difference between solid and fluid phases in a porous packed-bed. Intl J. Heat Mass Transfer 31, 173-177.

KuZnetsov, A. V. 1998 Thermal nonequilibrium forced convection in porous media. In Transport Phenomena in Porous Media (ed. D. B. Ingham \& I. Pop), pp. 103-129, Pergamon.

McKibisin, R. 2005 Modeling heat and mass transfer processes in geothermal systems. In Handbook of Porous Media, 2nd edition (ed. K. Vafai), pp. 545-571. CRC Press.

NiELD, D. A. 1998 Effects of local thermal nonequilibrium in steady convective processes in a saturated porous medium: Forced convection in a channel. J. Porous Media 1, 181-186.

Nield, D. A. \& Bejan, A. 2006 Convection in Porous Media, (3rd edition). Springer.

Nield, D. A., Kuznetsov, A. V. \& Xiong, M. 2002 Effect of local thermal non-equilibrium on thermally developing forced convection in a porous medium. Intl J. Heat Mass Transfer 45, 4949-4955.

Nouri-Borujerdi, A., Noghrehabadi, A. R. \& Rees, D. A. S. 2007 The effect of local thermal non-equilibrium on impulsive conduction in porous media. Intl J. Heat Mass Transfer 50, 3244-3249.

REES, D. A. S. 2003 Vertical free convective boundary-layer flow in a porous medium using a thermal nonequilibrium model: Elliptic effects. Zeit. Angew Math. Phys. 54, 437-448.

REES, D. A. S. 2007 Microscopic modelling of the two-temperature model for conduction in periodic and heterogeneous media. (In preparation).

ReEs, D. A. S. \& Pop, I. 2000 Vertical free convection boundary layer flow in a porous medium using a thermal nonequilibrium model. J. Porous Media 3, 31-44.

ReEs, D. A. S. \& Pop, I. 2005 Local thermal nonequilibrium in porous medium convection. In Transport Phenomena in Porous Media III (ed. D. B. Ingham \& I. Pop), pp. 147-173. Pergamon.

Schotting, R. \& Landman, A. J. 2004 Towards a physically based theory of high-concentrationgradient dispersion in porous media. In Emerging Technologies and Techniques in Porous Media, (ed. D. B. Ingham, A. Bejan, E. Mamut \& I. Pop), NATO Science Series Vol. 134, pp. 321-336.

Schumann, T. E. W. 1929 Heat transfer: A liquid flowing through a porous prism. J. Franklin Inst. 208, 405-416.

SHоOK, G. M. 2001 Predicting thermal breakthrough in heterogeneous media from tracer tests. Geothermics 30, 573-589.

Stopa, J. \& Wojnarowski, P. 2006 Analytical model of cold water front movement in a geothermal reservoir. Geothermics 35, 59-69.

StRaUghan, B. 2006 Global nonlinear stability in porous convection with a thermal non-equilibrium model. Proc. R. Soc. Lond. A 462, 409-418.

Swailes, D. C. \& PotTs, I. 2006 Transient heat transport in gas flow through granular porous media. Transp. Porous Media 65, 133-157.

TOOVEY, I. \& DAYAN, J. 1985 Analytical solution for the heat-transfer problem of fluid flowing through a packed-bed of porous solids. Trans. ASME J. Heat Transfer 107, 713-716.

VADASZ, P. 2005 Lack of oscillations in dual-phase-lagging heat conduction for a porous slab subject to imposed heat flux and temperature. Intl J. Heat Mass Transfer 48, 2822-2828.

VafaI, K. \& Amiri, A. 1998 Non-Darcy effects on confined forced convective flows. In Transport Phenomena in Porous Media I (ed. D. B. Ingham \& I. Pop), pp. 313-329. Pergamon.

WaKaO, N. \& KagueI, S. 1982 Heat and mass transfer in packed beds. Gordon and Breach. 